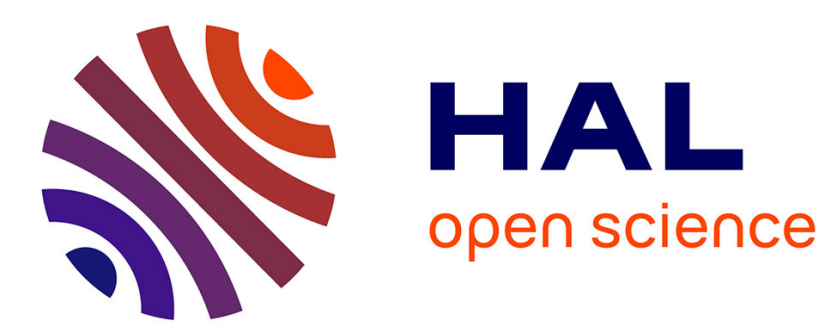

\title{
A nonlinear estimator for the activity of neuronal populations in the hippocampus
}

Michelle Chong, Romain Postoyan, Dragan Nesic, Levin Kuhlmann, Andrea Varsavsky

\section{- To cite this version:}

Michelle Chong, Romain Postoyan, Dragan Nesic, Levin Kuhlmann, Andrea Varsavsky. A nonlinear estimator for the activity of neuronal populations in the hippocampus. IFAC World Congress, 2011, Milan, Italy. pp.9899-9904, 10.3182/20110828-6-IT-1002.01844 • hal-00768749

\section{HAL Id: hal-00768749 \\ https://hal.science/hal-00768749}

Submitted on 23 Dec 2012

HAL is a multi-disciplinary open access archive for the deposit and dissemination of scientific research documents, whether they are published or not. The documents may come from teaching and research institutions in France or abroad, or from public or private research centers.
L'archive ouverte pluridisciplinaire HAL, est destinée au dépôt et à la diffusion de documents scientifiques de niveau recherche, publiés ou non, émanant des établissements d'enseignement et de recherche français ou étrangers, des laboratoires publics ou privés. 


\title{
A nonlinear estimator for the activity of neuronal populations in the hippocampus *
}

\author{
Michelle Chong* Romain Postoyan* Dragan Nešić* \\ Levin Kuhlmann ${ }^{*, * *}$ Andrea Varsavsky ${ }^{*, * * *}$ \\ * Department of Electrical and Electronics Engineering, The University \\ of Melbourne, Australia (e-mail: \{chongms, postoyan, dnesic, levink, \\ ava\}@unimelb.edu.au). \\ ** Department of Optometry and Vision Science, The University of \\ Melbourne, Australia. \\ *** Department of Otolaryngology, The University of Melbourne,
} Australia.

\begin{abstract}
We present an estimator design to reconstruct the mean membrane potential of individual neuronal populations from a single channel simulated electroencephalographic signal based on a model of the hippocampus. The robustness of the estimator against variations in the synaptic gains of the neuronal populations and disturbances in the input and measurement is studied. Our results are further illustrated in simulations.
\end{abstract}

Keywords: system state estimation; brain models; biomedical; robustness; stability.

\section{INTRODUCTION}

Epilepsy is a neurological disease that affects 50 million people worldwide. The randomness of unprovoked seizures severely affects the lives of those involved. In an effort to better manage recurrent unprovoked seizures, estimation methods have been developed with the aim of observing seizure-causing mechanisms and to detect seizures from electroencephalographic (EEG) data.

The task of detecting epileptic seizures has been investigated since the 1970s, starting with algorithms to classify epileptic and non-epileptic behaviour based on measures extracted from the EEG signal. Recent developments include works by Mirowski et al. [2009] and Kuhlmann et al. [2010]. While measure-based methods provide 'yes' or 'no' answers to the occurrence of seizures, these methods do not provide a detailed view of the seizure-causing mechanisms and only successfully predict the occurrence of seizures under restrictive conditions (Mormann et al. [2007]).

On the other hand, model-based approaches have been undertaken in developing estimation methods to gain insight into the underlying neuronal mechanisms, particularly during epileptic seizures. In a study by O'Sullivan-Greene et al. [2009], it was shown using a simple brain model of networked clocks with a single channel EEG output that its observability is dependant upon the position of the sensors on the brain (EEG electrodes). The authors concluded that strategically placed depth electrodes (e.g. in the hippocampus) as opposed to grid electrodes on the cortex will provide a greater chance of success for state estimation. This study justifies interest in developing estimation methods for a particular region of the brain. Most existing works in this direction are performed under

^ This work was carried out with support from the St. Vincent's Hospital (Melbourne) Research Endowment Fund. the stochastic framework. A recent study by Frogerais [2008] used stochastic filters to estimate the states and parameters of a model of neuronal populations in the hippocampus proposed by Wendling et al. [2002]. This is with the ultimate aim of tracking the transition of the brain from non-seizure to seizure state. In $\mathrm{Lu}$ and Lee [2010], the authors took a different approach in tracking this transition by observing the trajectories of the system's parameters and noting the region these parameters reside in. The regions correspond to polytopes representing the different EEG activities produced by the model proposed by Wendling et al. [2002]. However, several drawbacks of these studies include that the initial condition of the stochastic filter is required to be close to the true initial condition of the model, which is generally an unknown quantity, and that convergence is not guaranteed for every trajectory. We aim to address these issues in this study.

We propose a global exponential state estimator that reconstructs the mean membrane potential of individual neuronal populations in the hippocampus. We use a parameterised model proposed by Wendling et al. [2002] where a single-channel simulated EEG measurement is available. In the development of this estimator, we assume that the synaptic gains of each neuronal population (parameters) are known and we treat each parameter as constants during a particular brain activity due to its slow dynamics compared to the dynamics of the population's mean membrane potential. We also assume that the estimator is provided with known input as well as noisefree EEG measurement. We later relax these assumptions and show that the estimator is robust to uncertainty in parameters as well as disturbances in input and measurement within the input-to-state stability (ISS) framework of Sontag [1989]. The robustness of the proposed state estimator to parameter uncertainty provides a starting point in the final aim of building an estimator for both 
state and parameter of the model. This will form the further work of this study.

The paper is organised as follows: we define the notation in Section 2. In Section 3, we provide an overview of the model used. Section 4 discusses the assumptions used in the design of our proposed estimator in Section 5 . In Section 6, we show that the estimator is robust to parameter uncertainty as well as input and measurement disturbances. Simulation studies provided in Section 7 confirm our qualitative convergence and robustness analysis.

\section{NOTATION}

- We denote a vector $\left[\begin{array}{l}a \\ b\end{array}\right]$ as $(a, b)$, where $a, b \in \mathbb{R}$.

- The set $L_{\infty}$ denotes the set of functions $f: \mathbb{R} \rightarrow$ $\mathbb{R}^{n}$, for some $n \in \mathbb{Z}$, such that for any $0 \leq t_{1} \leq$ $t_{2}<\infty$ there exists $r \geq 0$ so that $\|f\|_{\left[t_{1}, t_{2}\right]}:=$ $\operatorname{ess} \sup _{\tau \in\left[t_{1}, t_{2}\right]}|f(\tau)| \leq r$.

\section{A MODEL OF NEURONAL POPULATIONS IN THE HIPPOCAMPUS}

We consider a model of neuronal populations in the hippocampus proposed by Wendling et al. [2002] as used in the development of estimation methods by Frogerais [2008], Lu and Lee [2010]. This model is capable of producing epileptic-like patterns in the EEG, typically observed in focal seizures that occur in the hippocampus. The interactions between four neuronal populations: the pyramidal neurons in the hippocampus, the local excitatory, fast inhibitory and slow inhibitory interneurons are described by the model. We write the model in this form:

$$
\begin{aligned}
& \dot{x}=A x+\Phi(x, \theta)+\sigma(u, y, \theta) \\
& y=C x,
\end{aligned}
$$

where the state vector is $x=\left(y_{0}, z_{0}, y_{1}, z_{1}, y_{2}, z_{2}, y_{3}, z_{3}\right.$, $\left.y_{4}, z_{4}\right) \in \mathbb{R}^{10}$, output $y \in \mathbb{R}$, input $u \in \mathbb{R}$ and parameter vector $\theta \in \mathbb{R}^{3}$. Each pair of state variables $\left(y_{i}, z_{i}\right)$ where $i=\{0, \ldots, 4\}$ represents the activity of the pyramidal neurons $(i=0)$, the excitatory interneurons $(i=1)$, the fast $(i=2)$, slow $(i=3)$ inhibitory interneurons and the interaction between the fast and slow inhibitory interneurons $(i=4)$ respectively. Physically, the variables $y_{1}, y_{2}$ and $y_{3}$ correspond to the contribution of the excitatory, fast and slow inhibitory populations to the mean membrane potential of the pyramidal neurons. A linear combination of these variables results in the EEG signal $y$ measured. The EEG signal here refers to measurements obtained using a depth electrode inserted in the hippocampus.

Matrix $A=\left[\begin{array}{ccccc}A_{0} & 0 & 0 & 0 & 0 \\ 0 & A_{1} & 0 & 0 & 0 \\ 0 & 0 & A_{2} & 0 & 0 \\ 0 & 0 & 0 & A_{3} & 0 \\ 0 & 0 & 0 & 0 & A_{4}\end{array}\right] \in \mathbb{R}^{10 \times 10}$ is Hurwitz with $A_{i}=\left[\begin{array}{cc}0 & 1 \\ -k_{i}^{2} & -2 k_{i}\end{array}\right]$, for $i=0,1,2,3,4$ with $k_{0}=k_{1}=a, k_{2}=g$ and $k_{3}=k_{4}=b$, where $a, b$ and $g$ are strictly positive constants, $C=$ $\left[\begin{array}{llllllllll}0 & 0 & 1 & 0 & -1 & 0 & -1 & 0 & 0 & 0\end{array}\right]$. The values and meanings of the constants used in this section are summarised in Appendix $\mathrm{B}$. We note that the pair $(C, A)$ is detectable, which is crucial in the design of the estimator. We denote $\Phi(x, \theta)=\left(0,0,0, \theta_{A} a C_{2} S\left(C_{1} y_{0}\right), 0, \theta_{G} g C_{7} S\left(C_{5} y_{0}-\right.\right.$ $\left.\left.C_{6} y_{4}\right), 0, \theta_{B} b C_{4} S\left(C_{3} y_{0}\right), 0, \theta_{B} b S\left(C_{3} y_{0}\right)\right)$ and $\sigma(y, u, \theta)=\left(0, \theta_{A} a S(y), 0, \theta_{A} a u, 0,0,0,0,0,0\right)$,

where $S(u)=\frac{\alpha}{1+e^{-r\left(u-V_{0}\right)}}, u \in \mathbb{R}$ is a sigmoid function that converts mean membrane potential to the mean firing rate of the neuronal population considered. Function $S$ is globally Lipschitz, i.e. there exists $\rho>0$ such that $|S(u)-S(v)| \leq \rho|u-v|$ for $u, v \in \mathbb{R}$ and is bounded: $S(z) \leq \alpha$, for all $z \in \mathbb{R}$.

The input $u \in \mathbb{R}$ represents all the external influences from afferent neural populations or structures (e.g. thalamus).

Three parameters $\theta_{A}, \theta_{B}$ and $\theta_{G}$ responsible for the generation of EEG signals depicting seizure and non-seizure activities were identified in Wendling et al. [2002]. These parameters correspond to the synaptic gains of the excitatory, slow and fast inhibitory populations respectively. The parameters form the vector $\theta=\left(\theta_{A}, \theta_{B}, \theta_{G}\right) \in \mathbb{R}^{3}$.

\section{PROBLEM STATEMENT AND ASSUMPTIONS}

The main purpose of this paper is to build a global exponential estimator for system (1). This is achieved based on several assumptions:

Assumption 1. The parameters $\theta$ (synaptic gain of each neuronal population) are constant and known.

These parameters are typically slowly-varying during a particular brain activity, such that they can be considered constant over the time period observed [Khalil, 2000, Section 9.6]. When the brain switches from one activity to another as identified in Wendling et al. [2002] however, the parameters will change. We further assume that the parameters have been identified. As no measuring method exists to verify the true parameter values, we take this into account by analysing the robustness of the estimator towards parameter uncertainty in Section 6 .

Assumption 2. The input $u$ belongs to $L_{\infty}$.

This assumption is justified since the input $u$ is a bounded signal in reality. In Wendling et al. [2002], the input is modelled as a white Gaussian noise with mean $90 \mathrm{mV}$ and variance $30 \mathrm{mV}$, that does satisfy Assumption 2 .

Assumption 3. The input $u$ is known.

The input to the brain is not quantifiable in practice, hence this assumption is unrealistic in reality. In Section 6 , we relax this assumption by allowing the input to be uncertain. We will show that good estimates can still be obtained provided that the $L_{\infty}$ norm of the difference between the true and assumed input is small.

Assumption 4. The measured EEG $y$ is noise-free.

The measured EEG considered here is recorded using depth electrodes. This ensures that the measurements are less noisy than recordings from scalp electrodes.

\section{A NONLINEAR ESTIMATOR}

The proposed estimator is of the form:

$$
\dot{\hat{x}}=A \hat{x}+\Phi(\hat{x}, \theta)+\sigma(u, y, \theta),
$$

where $\hat{x} \in \mathbb{R}^{10}$ is the state estimate. We note that the proposed estimator (2) is a copy of the model's dynamics (1). This usually results in an estimator that is sensitive 
to uncertainties, however we prove in Section 6 that our proposed estimator is robust to uncertainties in parameters as well as disturbances in the input and measurement. We also note that we cannot tune the convergence speed of the estimator as there is no observation gain. Nevertheless, simulations presented in Section 7 have shown us that the estimated state converges sufficiently fast to the true state.

Denoting the estimation error as $\tilde{x}:=x-\hat{x}$, we obtain the estimation error dynamics as

$$
\dot{\tilde{x}}=A \tilde{x}+\Phi(x, \theta)-\Phi(\hat{x}, \theta) .
$$

We prove in Theorem 1 using the cascade structure of system (3) that the proposed estimator ensures that the origin of the estimation error system (3) is globally exponentially stable (GES) [Khalil, 2000, Definition 4.5].

Theorem 1. Consider the model (1) and suppose Assumptions 1-4 hold. The origin of the estimation error system (3) is GES, i.e. there exist $k, \lambda>0$ such that

$$
|\tilde{x}(t)| \leq k e^{-\lambda t}|\tilde{x}(0)| \quad \forall t \geq 0, \forall \tilde{x}(0) \in \mathbb{R}^{10} .
$$

Proof. The proof is provided in Appendix A.1.

The exponential convergence property of the proposed estimator is desirable in practice as the estimates can be guaranteed to converge to the true states in exponential time. Additionally, the validity of the convergence property for any initial states is highly appreciated as the initial conditions are usually unknown.

Remark 1. [Rajamani, 1998, Theorem 2] provides a necessary and sufficient condition such that the estimation error asymptotically converges to the origin for a class of nonlinear observers that includes our proposed design. However, as system (1) is a parameterised family of systems with respect to $\theta$, this condition is difficult to verify. We have also tried to develop high gain observers (Raghavan and Hedrick [1994]) and circle criterion observers (Arcak and Kokotović [2001a,b]) for this model as the non-linearities are globally Lipschitz and monotonically non-decreasing. Our efforts included extending the result of Arcak and Kokotović [2001b] to account for a vector of non-linearities. These designs were unsuccessful due to feasibility issues faced when solving a linear matrix inequality.

\section{ROBUSTNESS ANALYSIS}

Some of the assumptions set out in Section 4 are not a good representation of practical situations, but they provided us with a good first step in designing the estimator. We now relax Assumptions 1, 3 and 4 to characterise their effects on the convergence property of the estimator.

Under realistic conditions, the parameters $\theta_{A}, \theta_{B}$ and $\theta_{G}$ of the model are not known, nor do they remain constant over time (see Section 4). We characterise these uncertainties in parameters by introducing bounded, time-varying signal $\epsilon_{\theta}(t)$ to the parameters of the model (1) as shown in $(4)$, so that the true parameter values in the model become $\theta+\epsilon_{\theta}$.

Similarly, we introduce bounded, time-varying disturbances $\epsilon_{u}(t)$ to the input available to the estimator and $\epsilon_{y}(t)$ to the measurement received by the estimator respectively as shown in (5). Thus, the real input to (1) is modelled by $u+\epsilon_{u}$, whereas the estimator is fed by an assumed input $u$. The introduction of $\epsilon_{u}$ relaxes Assumption 3 by allowing the real input to be unknown to the estimator. We introduce disturbance $\epsilon_{y}$ to characterise measurement noise. Therefore, we obtain the following perturbed systems (4) and (5) as illustrated in Figure 1:

$$
\begin{aligned}
& \left\{\begin{array}{l}
\dot{x}=A x+\Phi\left(x, \theta+\epsilon_{\theta}\right)+\sigma\left(u+\epsilon_{u}, y, \theta+\epsilon_{\theta}\right), \\
y=C x .
\end{array}\right. \\
& \dot{\hat{x}}=A \hat{x}+\Phi(\hat{x}, \theta)+\sigma\left(u, y+\epsilon_{y}, \theta\right) .
\end{aligned}
$$

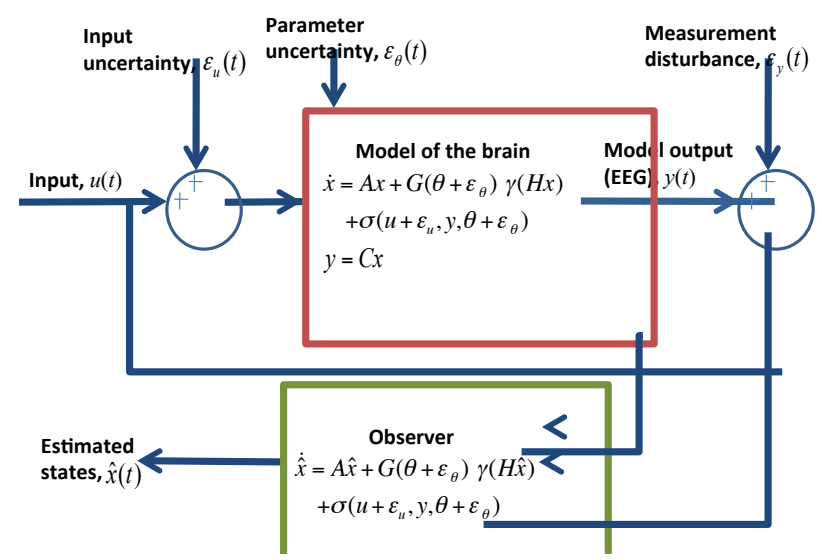

Fig. 1. Perturbed systems

We show in Theorem 2 that the the estimation error is input-to-state stable (ISS) (Sontag [1989]) with respect to $\epsilon_{u}, \epsilon_{y}$ and $\epsilon_{\theta}$.

Theorem 2. Consider the perturbed estimation error system (4)-(5) under Assumption 2. Then the estimator (5) guarantees for all $\tilde{x}(0) \in \mathbb{R}^{10}, t \geq 0$, and $\epsilon_{u}, \epsilon_{y}, \epsilon_{\theta} \in L_{\infty}$,

$$
|\tilde{x}(t)| \leq k e^{-\lambda t}|\tilde{x}(0)|+d_{1}\left\|\epsilon_{\theta}\right\|_{[0, t]}+d_{2}\left\|\epsilon_{y}\right\|_{[0, t]}+d_{3}\left\|\epsilon_{u}\right\|_{[0, t]},
$$

for constants $k, \lambda>0, d_{1}=\frac{k}{\lambda}\left|\left(a \alpha, a C_{2} \alpha, g C_{7} \alpha, b C_{4} \alpha, b \alpha\right)\right|$ $+\frac{k}{\lambda} a\|u\|_{[0, t]}, d_{2}=\frac{k}{\lambda} \theta_{A} a \rho$ and $d_{3}=\frac{k}{\lambda} \theta_{A} a$ where $\alpha, a, b, g$, $C_{2}, C_{4}, C_{7}$ and $\theta_{A}$ are constants defined in Section 3.

Proof. The proof is provided in Appendix A.2.

From Theorem 2, the estimation error exponentially converges to a neighbourhood of the origin whose size depends upon the $L_{\infty}$ norm of the perturbations $\epsilon_{\theta}, \epsilon_{y}$ and $\epsilon_{u}$. In that way, a small uncertainty in the input, output signals and parameters implies a small error on the state estimate. When all the perturbations are set to 0 , we recover the nominal estimation error system (3) that is GES as shown in Theorem 1.

\section{SIMULATION RESULTS}

We now illustrate the performance of the estimator derived in Section 5 using simulations.

The parameters $\theta_{A}, \theta_{B}$ and $\theta_{G}$ used correspond to seizure activity $\left(\theta_{A}=5, \theta_{B}=25\right.$ and $\left.\theta_{G}=10\right)$ identified in Wendling et al. [2002]. The initial conditions of the model (1) and the estimator (2) were set to $x(0)=$ $(6,6,6,6,6,6,6,6,6,6)$ and $\hat{x}(0)=(0,0,0,0,0,0,0,0,0,0)$. Simulations were performed with the constant values given in Appendix B. 


\subsection{Nominal case}

We first present simulations for the estimator under Assumptions 1-4. This is the ideal case where the parameters are constant and known as well as that input $u$ and measurement $y$ are known and unperturbed. Both model and estimator systems are supplied with the same Gaussian noise input with mean $90 \mathrm{mV}$ and variance $30 \mathrm{mV}$ used in Wendling et al. [2002]. As shown in Theorem 1 and illustrated in Figures 2 and 3, the state estimation error $\tilde{x}:=x-\hat{x}$ converges to 0 . We observe in simulations that the speed of convergence is in general faster than the duration of a particular brain activity (see Wendling et al. [2002]): by $t=0.9 \mathrm{~s}$, all state estimation errors have converged to 0 .
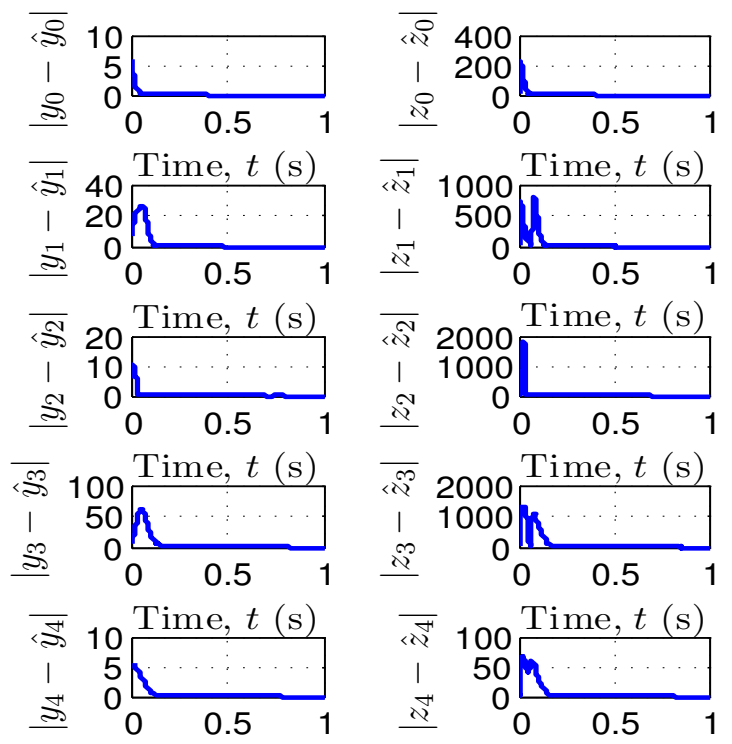

Time, $t(\mathrm{~s})$

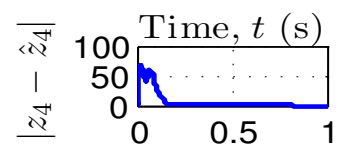

Time, $t(\mathrm{~s})$

Fig. 2. Nominal case: absolute state estimation error $\left|\tilde{x}_{i}\right|$ for $i \in\{0, \ldots, 4\}$.

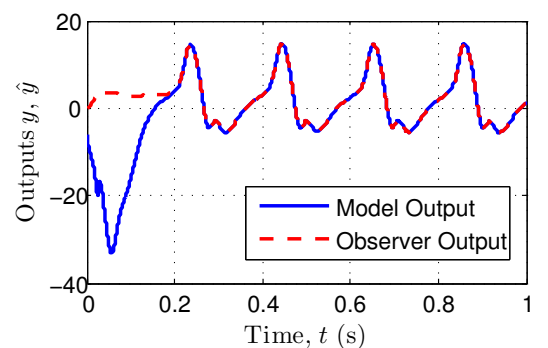

Fig. 3. Simulated EEG $y$ (solid line) and estimated EEG $\hat{y}$ (dashed line).

\subsection{Robustness}

Next, we test the performance of the estimator under uncertainties in the parameters $\theta_{A}, \theta_{B}$, and $\theta_{G}$ and disturbances in the input and output of the estimator.

Under the same conditions in Section 7.1, we simulate the perturbed systems (4) and (5) with constant perturbation values $\epsilon_{\theta}=(1,1,1), \epsilon_{u}$ is a Gaussian signal with mean
$0 \mathrm{mV}$ and standard deviation of $10 \mathrm{mV}$, and $\epsilon_{y}$ is a Gaussian signal with mean $0 \mathrm{mV}$ and standard deviation of $2 \mathrm{mV}$. Figure 4 shows that the estimation error converges to a neighbourhood of the origin. This observation agrees with Theorem 2.
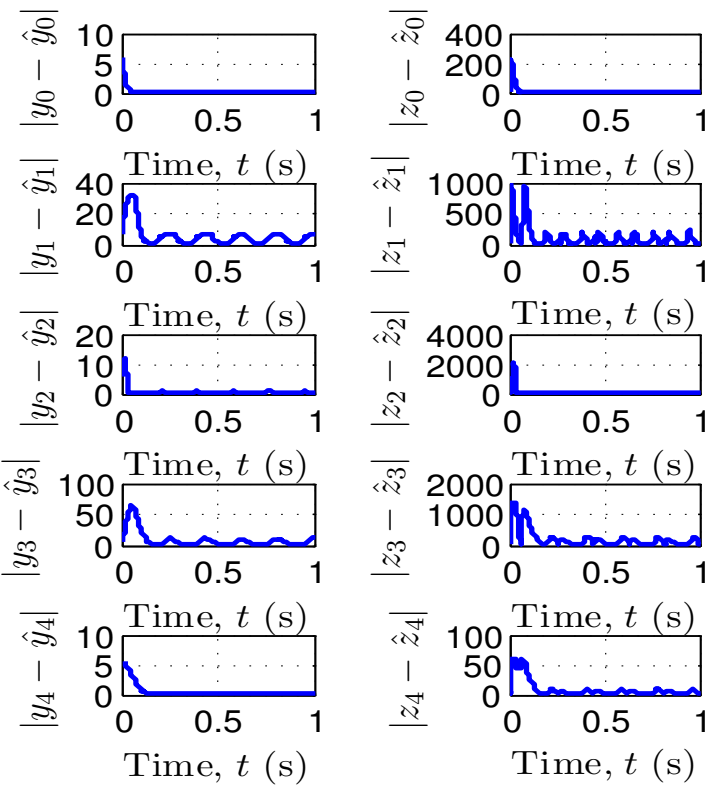

Fig. 4. Absolute state estimation error $\left|\tilde{x}_{i}\right|$ for $i \in$ $\{0, \ldots, 4\}$ under uncertainty in parameters of the model (1), as well as disturbances to the input and output of the estimator (2).

On the other hand, the input to the brain is hard to quantify under realistic conditions. To gauge the performance of the estimator under this condition, we perform another set of simulations where the input is set to $u=0$ for the estimator with no uncertainty in parameters and measurement noise. This case is qualitatively proven in Theorem 2 with $\epsilon_{\theta}=(0,0,0), \epsilon_{y}=0$ and $\epsilon_{u}(t)$ represents the real input received by the model (1). Figure 5 shows that the estimation error converges to a neighbourhood of the origin as expected. The estimation error for subsystems $\left(y_{0}, z_{0}\right),\left(y_{2}, z_{2}\right),\left(y_{3}, z_{3}\right)$ and $\left(y_{4}, z_{4}\right)$ show convergence to a reasonably small neighbourhood of the origin. However, the $\left(y_{1}, z_{1}\right)$ subsystem shows a much larger steady state estimation error than the other subsystems due to the input $u$ directly affecting it. This shows that the states can be reasonably reconstructed when the input is unknown.

\section{CONCLUSION}

We have successfully built an estimator that can reconstruct the mean membrane potential of the individual neuronal populations based on a model proposed by Wendling et al. [2002] using a simulated single channel EEG signal. The proposed estimator gives estimates that converge exponentially to the true states of the model. Also, the global convergence property of our proposed estimator ensures that any initial estimated state will converge to the true state of the model. This result is desirable because we do not know the initial state of the brain.

The proposed estimator is robust to variation in parameters and disturbances in the input and EEG measure- 


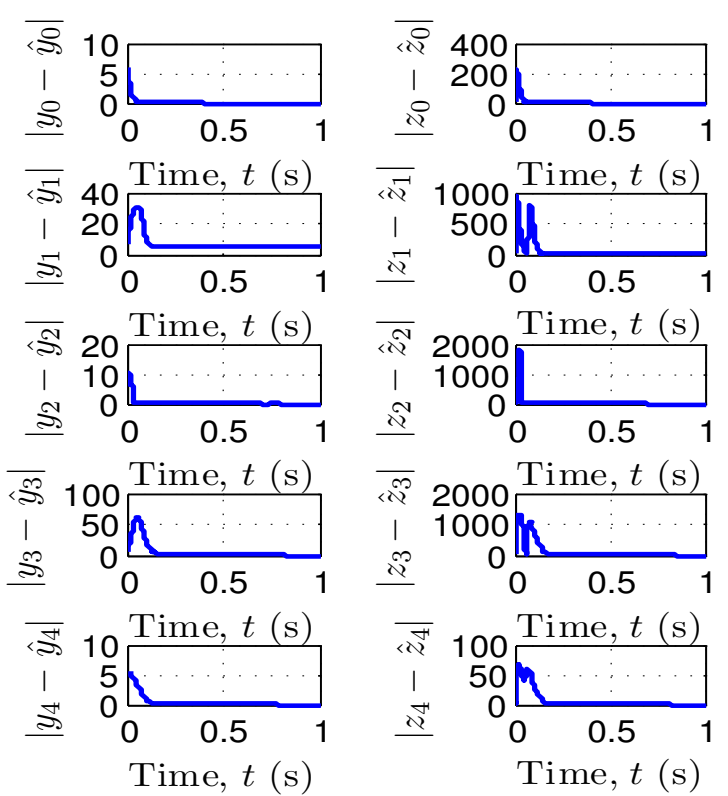

Fig. 5. Absolute state estimation error $\left|\tilde{x}_{i}\right|$ for $i \in$ $\{0, \ldots, 4\}$ with input to the estimator set to 0 .

ment in the sense that bounded perturbation will result in bounded estimation error. We have shown in simulations that the robustness of the estimator towards parameter, input and measurement perturbations provides us with good estimates of the actual membrane potential of neuronal populations under practical conditions.

Nevertheless, it has been identified in Wendling et al. [2002] that the variation in the synaptic gains of the individual neuronal populations $\theta_{A}, \theta_{B}$ and $\theta_{G}$ leads to different activities in the EEG. In order to provide early detection of seizure onsets, we will require the simultaneous estimation of both states and parameters of the model. The success in building a global exponential estimator provides hope for the design of an adaptive estimator to tackle the challenging problem of detecting and predicting epileptic seizures.

\section{REFERENCES}

M. Arcak and P. Kokotović. Nonlinear observers: a circle criterion design and robustness analysis. Automatica, 37 (12):1923-1930, 2001a.

M. Arcak and P. Kokotović. Observer-based control of systems with slope-restricted nonlinearities. IEEE Transactions on Automatic Control, 46:1, $2001 \mathrm{~b}$.

P. Frogerais. Model and identification in epilepsy: from neuronal population dynamics to EEG signals (in French). PhD thesis, University of Rennes 1, 2008.

H.K. Khalil. Nonlinear systems. Prentice Hall, 3rd edition, 2000.

L. Kuhlmann, D. Freestone, A. Lai, A.N. Burkitt, K. Fuller, D.B. Grayden, L. Seiderer, S. Vogrin, I.M.Y. Mareels, and M.J. Cook. Patient-specific bivariatesynchrony-based seizure prediction for short prediction horizons. Epilepsy Research, 2010.

T. Lu and J. Lee. Polytopic linear parameter-varying model of epileptiform activity. In American Control Conference, Baltimore, U.S.A., 2010.
P. Mirowski, D. Madhavan, Y. LeCun, and R. Kuzniecky. Classification of patterns of EEG synchronization for seizure prediction. Clinical neurophysiology, 120(11): 1927-1940, 2009.

F. Mormann, R.G. Andrzejak, C.E. Elger, and K. Lehnertz. Seizure prediction: the long and winding road. Brain, 130(2):314, 2007.

E. O'Sullivan-Greene, I. Mareels, A. Burkitt, and L. Kulhmann. Observability issues in networked clocks with applications to epilepsy. In IEEE Conference on Decision and Control, Shanghai, China, 2009.

S. Raghavan and J.K. Hedrick. Observer design for a class of nonlinear systems. International Journal of Control, 59(2):515-528, 1994.

R. Rajamani. Observers for Lipschitz nonlinear systems. IEEE Transactions on Automatic Control, 43(3):397401, 1998.

E.D. Sontag. Smooth stabilization implies coprime factorization. IEEE Transactions on Automatic Control, 34 (4):435-443, 1989.

F. Wendling, F. Bartolomei, J.J. Bellanger, and P. Chauvel. Epileptic fast activity can be explained by a model of impaired GABAergic dendritic inhibition. European Journal of Neuroscience, 15(9):1499-1508, 2002.

\section{Appendix A. PROOFS}

\section{A.1 Proof of Theorem 1}

The proof relies on the fact that we can decompose system (3) into 5 subsystems $\Sigma_{0}$ to $\Sigma_{4}$ in a cascaded form.

The five subsystems $\Sigma_{0}, \Sigma_{1}, \Sigma_{2}, \Sigma_{3}$ and $\Sigma_{4}$ are

$$
\begin{array}{ll}
\Sigma_{0}: & \dot{\tilde{x}}_{0}=A_{0} \tilde{x}_{0} \\
\Sigma_{1}: & \dot{\tilde{x}}_{1}=A_{1} \tilde{x}_{1}+\phi_{1}\left(y_{0}\right)-\phi_{1}\left(\hat{y}_{0}\right) \\
\Sigma_{2}: & \dot{\tilde{x}}_{2}=A_{2} \tilde{x}_{2}+\phi_{2}\left(y_{0}, y_{4}\right)-\phi_{2}\left(\hat{y}_{0}, \hat{y}_{4}\right) \\
\Sigma_{3}: & \dot{\tilde{x}}_{3}=A_{3} \tilde{x}_{3}+\phi_{3}\left(y_{0}\right)-\phi_{3}\left(\hat{y}_{0}\right) \\
\Sigma_{4}: & \dot{\tilde{x}}_{4}=A_{4} \tilde{x}_{4}+\phi_{4}\left(y_{0}\right)-\phi_{4}\left(\hat{y}_{0}\right),
\end{array}
$$

where $\tilde{x}_{i}:=\left(\tilde{x}_{i 1}, \tilde{x}_{i 2}\right)=\left(y_{i}-\hat{y}_{i}, z_{i}-\hat{z}_{i}\right) \in \mathbb{R}^{2}$ for $i=0,1,2,3,4$. $\phi_{1}\left(y_{0}\right)=\left(0, \theta_{A} a C_{2} S\left(C_{1} y_{0}\right)\right), \phi_{2}\left(y_{0}, y_{4}\right)=$ $\left(0, \theta_{G} g S\left(C_{5} y_{0}-C_{6} y_{4}\right)\right), \phi_{3}\left(y_{0}\right)=\left(0, \theta_{B} b C_{4} S\left(C_{3} y_{0}\right)\right)$ and $\phi_{4}\left(y_{0}\right)=\left(0, \theta_{B} b S\left(C_{3} y_{0}\right)\right)$. Matrices $A_{0}, A_{1}, A_{2}, A_{3}, A_{4}$ are Hurwitz as defined in Section 3.

First note that systems (1) and (2) are forward complete due to the global Lipschitz property of function $S$ [Khalil, 2000, Theorem 3.2] and input $u \in L_{\infty}$. Consequently, we can show that (3) is forward complete.

We have broken the proof down to 3 parts. In Part 1, we will show that $\Sigma_{0}$ is GES. We will then show that system $\Sigma_{0}-\Sigma_{1}-\Sigma_{3}-\Sigma_{4}$ is GES in Part 2. Finally in Part 3, we will show that system (3) is GES.

Part 1: As $A_{0}$ is Hurwitz, $\Sigma_{0}$ is GES.

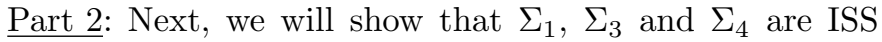
with respect to $\tilde{x}_{0}$.

For all $t \geq 0, \tilde{x}_{1}(0) \in \mathbb{R}^{2}$ and $y_{0}, \hat{y}_{0} \in L_{\infty}$, the analytic solution for subsystem $\Sigma_{1}$ is

$$
\begin{aligned}
\tilde{x}_{1}(t)= & e^{A_{1} t} \tilde{x}_{1}(0) \\
& +\int_{0}^{t} e^{A_{1}(t-s)}\left(\phi_{1}\left(y_{0}(s)\right)-\phi_{1}\left(\hat{y}_{0}(s)\right)\right) d s .
\end{aligned}
$$


Therefore,

$$
\begin{aligned}
& \left|\tilde{x}_{1}(t)\right| \leq\left|e^{A_{1} t}\right|\left|\tilde{x}_{1}(0)\right|+\int_{0}^{t}\left|e^{A_{2}(t-s)}\right| \\
& \times\left|\theta_{A} a C_{2}\left(S\left(C_{1} y_{0}(s)\right)-S\left(C_{1} \hat{y}_{0}(s)\right)\right)\right| d s .
\end{aligned}
$$

Since $A_{1}$ is Hurwitz, there exists $k_{1}, \lambda_{1} \geq 0$ such that $\left|e^{A_{1} t}\right| \leq k_{1} e^{-\lambda_{1} t}$ and since the function $S$ is globally Lipschitz with constant $\rho$, we have that:

$$
\begin{aligned}
\left|\tilde{x}_{1}(t)\right| \leq & k_{1} e^{-\lambda_{1} t}\left|\tilde{x}_{1}(0)\right| \\
& +\int_{0}^{t}\left|e^{A_{1}(t-s)}\right| \theta_{A} a C_{2} \rho C_{1}\left|y_{0}(s)-\hat{y}_{0}(s)\right| d s \\
\leq & k_{1} e^{-\lambda_{1} t}\left|\tilde{x}_{1}(0)\right|+\rho_{1}\left\|\tilde{x}_{01}\right\|_{[0, t]} \int_{0}^{t}\left|e^{A_{1}(t-s)}\right| d s
\end{aligned}
$$

where $\rho_{1}=\theta_{A} a C_{2} \rho C_{1}$. Also $\int_{0}^{t}\left|e^{A_{1}(t-s)}\right| d s \leq k_{1}$ $\times \int_{0}^{t} e^{-\lambda_{1}(t-s)} d s \leq \frac{k_{1}}{\lambda_{1}}\left(1-e^{-\lambda_{1} t}\right) \leq \frac{k_{1}}{\lambda_{1}}$. Hence $\left|\tilde{x}_{1}(t)\right| \leq$ $k_{1} e^{-\lambda_{1} t}\left|\tilde{x}_{1}(0)\right|+\rho_{1} \frac{k_{1}}{\lambda_{1}}\left\|\tilde{x}_{01}\right\|_{[0, t]}$. Therefore, subsystem $\Sigma_{1}$ with input $\tilde{x}_{01}$ is ISS with respect to $\tilde{x}_{01}$.

Similarly for $\Sigma_{3}$ and $\Sigma_{4}$, we can show that $\left|\tilde{x}_{3}(t)\right| \leq$ $k_{3} e^{-\lambda_{3} t}\left|\tilde{x}_{3}(0)\right|+\rho_{3} \frac{k_{3}}{\lambda_{3}}\left\|\tilde{x}_{01}\right\|_{[0, t]}$ and $\left|\tilde{x}_{4}(t)\right| \leq k_{4} e^{-\lambda_{4} t}\left|\tilde{x}_{4}(0)\right|$ $+\rho_{4} \frac{k_{4}}{\lambda_{4}}\left\|\tilde{x}_{01}\right\|_{[0, t]}$ with constants $k_{3}, k_{4}, \lambda_{3}, \lambda_{4} \geq 0, \rho_{3}=$ $\theta_{B} b C_{4} \rho C_{3}$ and $\rho_{4}=\theta_{B} b C_{3} \rho$. Hence, $\Sigma_{1}, \Sigma_{3}$ and $\Sigma_{4}$ are ISS with respect to $\tilde{x}_{01}$. As $\Sigma_{0}$ is in cascade with $\Sigma_{1}, \Sigma_{3}$ and $\Sigma_{4}$, by [Khalil, 2000, Lemma 4.7 ${ }^{1}$, the cascaded system $\Sigma_{0}-\Sigma_{1}-\Sigma_{3}-\Sigma_{4}$ is GES.

$\underline{\text { Part 3: }}$ Similar to Part 2, we can show that for all $t \geq$ $0, \tilde{x}_{2}(0) \in \mathbb{R}^{2}$ and inputs to the subsystem $\tilde{x}_{01}, \tilde{x}_{41} \in L_{\infty}$,

$$
\left|\tilde{x}_{2}(t)\right| \leq k_{2} e^{-\lambda_{2} t}\left|\tilde{x}_{2}(0)\right|+\rho_{2} \frac{k_{2}}{\lambda_{2}}\left\|\left(\tilde{x}_{01}, \tilde{x}_{41}\right)\right\|_{[0, t]},
$$

where $k_{2}, \lambda_{2}>0, \rho_{2}=\theta_{G} g C_{7} \rho\left|\left(C_{5}, C_{6}\right)\right|$. Therefore, $\Sigma_{2}$ is ISS with respect to inputs $\tilde{x}_{01}$ and $\tilde{x}_{41}$. As $\Sigma_{2}$ is in cascade with $\Sigma_{0}-\Sigma_{1}-\Sigma_{3}-\Sigma_{4}$, the cascaded system (3) is GES [Khalil, 2000, Lemma 4.7].

\section{A.2 Proof of Theorem 2}

From (4) and (5), the perturbed error system is:

$$
\begin{aligned}
\dot{\tilde{x}}= & A \tilde{x}+\Phi\left(x, \theta+\epsilon_{\theta}\right)-\Phi(\hat{x}, \theta)+\sigma\left(u, y, \theta+\epsilon_{\theta}\right) \\
& -\sigma\left(u+\epsilon_{u}, y+\epsilon_{y}, \theta\right) \\
= & A \tilde{x}+\Psi_{n o m}(x, \hat{x})+\Psi_{y}\left(y, \epsilon_{y}\right) \\
& +\Psi_{\theta}\left(x, y, u, \epsilon_{\theta}\right)+\Psi_{u}\left(\epsilon_{u}\right),
\end{aligned}
$$

where $\Psi_{n o m}(x, \hat{x})=\left(0,0,0, \theta_{A} a C_{2}\left\{S\left(C_{1} y_{0}\right)-S\left(C_{1} \hat{y}_{0}\right)\right\}, 0\right.$, $\theta_{G} g C_{7} S\left(C_{5} y_{0}-C_{6} y_{4}\right)-S\left(C_{5} \hat{y}_{0}-C_{6} \hat{y}_{4}\right), 0$,

$\left.\theta_{B} b C_{4}\left\{S\left(C_{3} y_{0}\right)-S\left(C_{3} \hat{y}_{0}\right)\right\}, 0, \theta_{B} b\left\{S\left(C_{3} y_{0}\right)-S\left(C_{3} \hat{y}_{0}\right)\right\}\right)$,

$\Psi_{\theta}\left(x, y, u, \epsilon_{\theta}\right)=\left(0, \epsilon_{\theta} a S(y), 0, \epsilon_{\theta} a C_{2} S\left(C_{1} y_{0}\right)+\epsilon_{\theta} a u, 0\right.$, $\left.\epsilon_{\theta} g C_{7} S\left(C_{5} y_{0}-C_{6} y_{4}\right), 0, \epsilon_{\theta} b C_{4} S\left(C_{3} y_{0}\right), 0, \epsilon_{\theta} b S\left(C_{3} y_{0}\right)\right)$,

$\Psi_{y}\left(y, \epsilon_{y}\right)=\left(0, \theta_{A} a\left\{S(y)-S\left(y+\epsilon_{y}\right)\right\}, 0,0,0,0,0,0,0,0\right)$ and $\Psi_{u}\left(\epsilon_{u}\right)=\left(0,0,0, \theta_{A} a \epsilon_{u}, 0,0,0,0,0,0\right)$.

1 [Khalil, 2000, Lemma 4.7] shows that the cascade of an ISS system with a globally asymptotically stable (GAS) system gives an overall GAS system. In the case where the KL-functions are exponential-KL (i.e $\beta(s, t)=k e^{-\lambda t} s$ where there exists $k, \lambda>0$ for all $s, t \geq 0$ ) and the ISS gain is a linear function, it can be similarly shown that this yields an overall GES system.
For all $t \geq 0, \tilde{x}(0) \in \mathbb{R}^{10}$, the solution of (A.1) is:

$$
\begin{aligned}
\tilde{x}(t)= & e^{A t} \tilde{x}(0)+\int_{0}^{t} e^{A(t-s)} \Psi_{n o m}(x(s), \hat{x}(s)) d s \\
& +\int_{0}^{t} e^{A(t-s)} \Psi_{\theta}\left(x(s), y(s), u(s), \epsilon_{\theta}(s)\right) d s \\
& +\int_{0}^{t} e^{A(t-s)} \Psi_{y}\left(y(s), \epsilon_{y}(s)\right) d s \\
& +\int_{0}^{t} e^{A(t-s)} \Psi_{u}\left(\epsilon_{u}(s)\right) d s .
\end{aligned}
$$

\begin{tabular}{|c|c|c|}
\hline Parameter & Description & Standard value \\
\hline$\frac{1}{a}$ & $\begin{array}{l}\text { Average time constant of the } \\
\text { excitatory loop. }\end{array}$ & $a=100 s^{-1}$ \\
\hline$\frac{1}{b}$ & $\begin{array}{l}\text { Average time constant of the } \\
\text { slow inhibitory loop. }\end{array}$ & $b=50 s^{-1}$ \\
\hline$\frac{1}{g}$ & $\begin{array}{l}\text { Average time constant of the } \\
\text { fast inhibitory loop. }\end{array}$ & $g=500 s^{-1}$ \\
\hline$V_{0}, \alpha, r$ & $\begin{array}{l}\text { Parameters for the sigmoid } \\
\text { function. } \alpha \text { is the maximum fir- } \\
\text { ing rate. } r \text { is the slope of the } \\
\text { sigmoid and } V_{0} \text { is the threshold } \\
\text { of the population's mean mem- } \\
\text { brane potential. }\end{array}$ & $\begin{array}{l}V_{0}=6 \mathrm{mV} \\
\alpha=5 \mathrm{~s}^{-1}, r= \\
0.56 \mathrm{mV}^{-1}\end{array}$ \\
\hline$C_{1}, C_{2}$ & $\begin{array}{l}\text { Average number of synaptic } \\
\text { contacts in the excitatory feed- } \\
\text { back loop }\end{array}$ & $\begin{array}{l}\text { With } C=135, \\
C_{1}=C \text { and } \\
C_{2}=0.8 C\end{array}$ \\
\hline$C_{3}, C_{4}$ & $\begin{array}{l}\text { Average number of synaptic } \\
\text { contacts in the slow feedback } \\
\text { inhibitory loop }\end{array}$ & $\begin{array}{l}C_{3}=C_{4}= \\
0.25 C\end{array}$ \\
\hline$C_{5}, C_{6}$ & $\begin{array}{l}\text { Average number of synaptic } \\
\text { contacts in the fast feedback } \\
\text { inhibitory loop }\end{array}$ & $\begin{array}{l}C_{5}=0.3 C \text { and } \\
C_{6}=0.1 C\end{array}$ \\
\hline$C_{7}$ & $\begin{array}{l}\text { Average number of synaptic } \\
\text { contacts between slow and fast } \\
\text { inhibitory interneurons }\end{array}$ & $C_{7}=0.8 C$ \\
\hline
\end{tabular}

From Theorem 1, we know that the nominal system (3) is GES, i.e. we have shown that $\mid e^{A t} \tilde{x}(0)+\int_{0}^{t} e^{A(t-s)}$ $\times \Psi_{\text {nom }}(x(s), \hat{x}(s)) d s \mid \leq k e^{-\lambda t}$ for constants $k, \lambda>0$. Moreover, $S$ is globally Lipschitz and $S(z) \leq \alpha$, for any $z \in \mathbb{R}$. Therefore,

$$
\begin{aligned}
|\tilde{x}(t)| \leq & k e^{-\lambda t}|\tilde{x}(0)|+(\bar{M}+\bar{N}) \int_{0}^{t}\left|e^{A(t-s)}\right|\left|\epsilon_{\theta}(s)\right| d s \\
& +\bar{K} \int_{0}^{t}\left|e^{A(t-s)}\right|\left|\epsilon_{y}(s)\right| d s \\
& +\bar{L} \int_{0}^{t}\left|e^{A(t-s)}\right|\left|\epsilon_{u}(s)\right| d s \\
\leq & k e^{-\lambda t}|\tilde{x}(0)|+(\bar{M}+\bar{N}) \frac{k}{\lambda}\left\|\epsilon_{\theta}\right\|_{[0, t]} \\
& +\bar{K} \frac{k}{\lambda}\left\|\epsilon_{y}\right\|_{[0, t]}+\bar{L} \frac{k}{\lambda}\left\|\epsilon_{u}\right\|_{[0, t]},
\end{aligned}
$$

where $\bar{M}=\left|\left(a \alpha, a C_{2} \alpha, g C_{7} \alpha, b C_{4} \alpha, b \alpha\right)\right|, \bar{N}=a\|u\|_{[0, t]}$, $\bar{K}=\theta_{A} a \rho$ and $\bar{L}=\theta_{A} a$. We obtain the desired results as in Theorem 2 by taking $d_{1}=\frac{k}{\lambda}(\bar{M}+\bar{N}), d_{2}=\frac{k}{\lambda} \bar{K}$ and $d_{3}=\frac{k}{\lambda} \bar{L}$.

\section{Appendix B. VALUES AND DESCRIPTION OF THE CONSTANTS USED ESTABLISHED IN WENDLING ET. AL. [2002]}

